\title{
Diffusion of coloured silica nanoparticles into human hair
}

\author{
Sandra Sampaio, ${ }^{a}$ Frederico Maia ${ }^{a, b}$ and Jaime R Gomes ${ }^{a, *}$ \\ ${ }^{a}$ Departamento de Engenharia Têxtil, 2C2T - Centro de Ciência e Tecnologia Têxtil, \\ Universidade do Minho, Portugal \\ Email:rgomes@det.uminho.pt \\ ${ }^{b}$ Ecoticket, Lda, Portugal
}

Received: 31 July 2010; Accepted: 3 September 2010

\begin{abstract}
A novel method for dyeing human hair fibres using coloured silica nanoparticles of $206 \mathrm{~nm}$ has been developed. Diffusion of coloured silica nanoparticles into hair fibres has been investigated; silica nanoparticles do not penetrate at all, or do so only sparingly, into the structure of Caucasian virgin hair. However, coloured silica nanoparticles diffused readily into bleached hair fibres. Scanning electron microscopy showed that coloured silica nanoparticles were present mainly in the outer regions of the hair structure (the inner layers of the cuticle and the outer region of the cortex). It was found that the silica nanoparticles were physically entrapped in the hair fibres and results showed a good shampoo fastness to washing.
\end{abstract}

\section{Introduction}

The present work is a continuation of the work, which started on wool and has moved on to other protein fibres such as hair [1], leading to a novel method of dyeing human hair fibres using coloured silica nanoparticles as an alternative to colouring hair with dyes. At present, hair dyeing is mainly a process using oxidation dyes that require developing with hydrogen peroxide. What we propose is a process that does not need developing, being a direct dyeing process.

Coloured silica nanoparticles combine the versatility and functionality of dyes with the stability and biocompatibility of the silica surface. Silica nanoparticles were synthesised using a modification of the Stöber $[2,7,8]$ synthesis method. Using appropriate synthetic conditions and by careful selection of dyes, a large number of dye molecules can be incorporated inside a single silica particle. For greater fastness, they were made to react with a linking agent, such as GLYMO, the amino group of the dye reacting with the glycidil group of GLYMO [3]. Moreover, as the dye is linked to the silica nanoparticles, keeping the dye from the surrounding environment, allergies that are sometimes caused by conventional dyes can be minimised. In this study, Fourier Transform-infrared (FTIR) spectroscopy, transmission electron microscopy (TEM) and scanning electron microscopy (SEM) were used to obtain information about the size of the coloured silica nanoparticles, as well as their dyeing performance on hair fibres.

To study the diffusion of the coloured silica nanoparticles, the complex morphological structure of the hair fibres has to be taken into consideration. For dyes, it determines the pathway that they can take during dyeing and is of critical importance for the rate and extent of the dye uptake [4]. The cuticle and epicuticle play an important role in controlling the diffusion rate of dyes and other molecules into the fibre. The condition of the hair affects the uptake and the diffusion of external substances to the inside. A rule of thumb is that diffusion is faster into altered or damaged hair than into unaltered hair [5].
Natural hair colour is as a result of melanine clusters of ca. $300 \mathrm{~nm}$ dispersed within the colourless keratin-based cortex of hair. Morphologically, a hair shaft contains three principal regions: the cuticle, the cortex and the medulla. Apart from the cuticle, the other major structural component of hair is the cortex. The cortex is the main bulk of the fibre and the cuticle is the outermost layer that protects the cortex. The cuticle consists of flat overlapping cells (scales), each cuticle cell is $0.3-0.5 \mu \mathrm{m}$ thick and the visible length is anywhere between 5 and $10 \mu \mathrm{m}$. In human hair, the cuticle layer is generally approximately 10 scales thick. Each cuticle cell consists of sublamellar layers: the epicuticle, A layer, exocuticle, endocuticle and cell membrane complex. The epicuticle is the cell membrane of the cuticle cell; the hydrophobic character of the epicuticle is attributable to the presence of lipids, predominantly 18-methyleicosanoic acid, covalently bound to the underlying proteins via thioester linkages with cysteine and the lipid layer attached to the epicuticle contributes to the lubricity of hair. The A layer and the exocuticle are rich in cystine content $(\sim 30 \%$ and $\sim 15 \%$, respectively), highly crosslinked and mechanically strong. The endocuticle is low in cystine content $(\sim 3 \%)$ and weak mechanically [6]. The cell membrane complex is the intercellular cement that holds the cuticle cells together. It is again a lamellar structure, with its intercellular material soft and characterised by a great potential for swelling in aqueous solutions and it is considered to be an important penetration pathway [7].

\section{Experimental}

\section{Materials}

Caucasian dark brown hair (without any chemicals) was generously supplied by volunteers. The ether petroleum was obtained from Riedel-de-Haen (Germany), ethanol was acquired from AGA (Portugal) and acetic acid from Sigma-Aldrich (USA), the benzyl alcohol was supplied by Panreac (Spain), the nonionic surfactant triton X-100 was 
acquired from Merck (), the silane crosslinker (3glycidoxypropyltrimethoxysilane, GLYMO) was supplied from Fluka and the Telon Red M-BL acid dye was supplied by Dystar. The salts, sodium metasilicate and ammonium chloride, were supplied by $\mathrm{BDH}$. The bleaching cream as an oxidising agent (a bleaching agent) was supplied by Frival Professional. The bleaching cream consists of two components and, when the two components are mixed, is converted into 6\% weight of hydrogen peroxide concentration and $\mathrm{pH}$ 10.5. Also, other active ingredients in the bleaching cream that aid in bleaching are potassium persulphate, ammonium persulphate and sodium persulphate. All products were applied as received, without any previous purification.

\section{Nanoparticles preparation and characterisation}

In this work, silica nanoparticles were obtained from a typical sol-gel reverse emulsion (W/O) with an aqueous dye solution. The process was an adaptation of the Stöber method [3,8]. First, one solution of sodium metasilicate was prepared $\left(1.3 \mathrm{~mol} \mathrm{l}^{-3}\right)$ and added to the solvent (100 $\mathrm{ml}$ of ether petroleum) under constant agitation with a stirrer, in order to form a sol-gel. Then, to the sol-gel was added $10 \mathrm{ml}$ of an aqueous solution (1\%) of Telon Red M-BL acid dye mixed with $10 \mathrm{ml}$ of GLYMO (freshly prepared). Finally, $20 \mathrm{ml}$ of $3.7 \mathrm{~mol} \mathrm{l}^{-3}$ ammonium chloride solution was added to precipitate the nanoparticles. The solvent was evaporated and the coloured silica nanoparticles were washed to remove the free dye from the suspension. The dye was fixed to the nanoparticles with a silane crosslinker by first adding an aqueous solution of dye to GLYMO, allowing the dye to react with the GLYMO at room temperature for $15 \mathrm{~min}$ and then adding the dye-silane complex to the sol-gel [3]. The coloured nanoparticles were characterised by transmission electron microscopy, dynamic light scattering and by Fourier Transforminfrared spectroscopy. Transmission electron microscopy images were obtained through a JEOL JEM-2010 transmission electron microscope. The particle size distribution was obtained by dynamic light scattering (DLS) using a Malvern Zetasizer NS (UK). Fourier Transform-infrared (FTIR) spectra were obtained as $\mathrm{KBr}$ pellets in the range $400-4000 \mathrm{~cm}^{-1}$ using an Avatar 360 FTIR spectrophotometer (Nicolet, USA).

\section{Hair bleaching}

Virgin Caucasian dark brown hair samples were treated with a lightening powder mixed with peroxide cream, both from Frival Professional, at a ratio of $1: 2$ (6\% wt hydrogen peroxide concentration) at $40{ }^{\circ} \mathrm{C}, \mathrm{pH} 10.5$ for $30 \mathrm{~min}$ at a hair-cream ratio of 1:10. The samples were then extensively washed in distilled water and dried at room temperature.

\section{Hair dyeing}

Bleached hair samples were dyed in a solution containing $10 \mathrm{~g} \mathrm{l}^{-1}$ of nonionic surfactant, $40 \mathrm{~g} \mathrm{l}^{-1}$ of the coloured silica nanoparticles colloidal suspension and $10 \%$ of benzyl alcohol or 5/5 (\% v/v) benzyl alcohol/ethanol. The hair coloured silica nanoparticle dyeing experiments (7.5\% owf dye) were performed at $\mathrm{pH} 4.5$ (adjusted with acetic acid) at $45{ }^{\circ} \mathrm{C}$ for $1 \mathrm{~h}$ and were carried out in a Linitest (Heraeus, USA) at a low liquor ratio (1:5). Hair samples dyed with coloured silica nanoparticles were rinsed with running water for $5 \mathrm{~min}$ and then dried at room temperature.

\section{Colour fastness to shampooing}

This assessment was aimed to evaluate the colour fastness property of dyed hair with respect to the daily washing process. For washing the hair, $200 \mathrm{ml}(0.2 \%)$ of a cleansing shampoo (Elvive Shampoo Color-Vive; L'Oreal, France) was used and the hair samples were washed in a Linitest machine for 2 min using a stainless steel container. The temperature was maintained at $40{ }^{\circ} \mathrm{C}$ and 20 plastic balls (5 $\mathrm{mm}$ in diameter) were added to simulate the hair washing. After shampoo washing, the hair samples were rinsed again with running water for 2 min. A finisher (Elvive Crème Amaciador Protector Color-Vive; L'Oreal) was then applied evenly to the hair samples for $5 \mathrm{~min}$ after the shampooing of the coloured hair. Finally, hair samples were furthered rinsed with $200 \mathrm{ml}$ of water for $2 \mathrm{~min}$ and then dried at room temperature. The colour fastness to shampooing was tested up to 13 washings.

\section{Instrumental assessment of colour fastness to shampooing}

Colour changes were measured in terms of $L^{*}, a^{*}$ and $b^{*}$ CIELAB coordinates using a Datacolor SF600 instrument, at D65 daylight with $10^{\circ}$ standard observer, before and after hair washes. Difference in colour $(\Delta E)$ was calculated using the following equation to evaluate colour change before and after washes: $\Delta E=$ $\left[\left(L_{t}-L_{o}\right)^{2}+\left(a_{t}-a_{o}\right)^{2}+\left(b_{t}-b_{o}\right)^{2}\right]^{1 / 2}$. The lower the $\Delta E$ values, the better the colour fastness of the hair samples will be.

\section{Scanning electron microscopy}

Scanning electron microscopy scans of the surface and cross section of the virgin, bleached hair dyed with the coloured silica nanoparticles were taken using the LeicaCambridge S360 scanning electron microscope and the NanoSEM-FEI Nova 200 (FEG/SEM). Cross-section pictures of the hair fibres were taken after embedding the hair into a low-viscosity epoxy resin at room temperature for $24 \mathrm{~h}$ (cure time) and, after hand cutting, those hair fibres were immersed in liquid nitrogen.

\section{Results and Discussion}

\section{Nanoparticle characterisation}

A colloidal suspension of coloured nanoparticles was obtained. The FTIR data suggest that the dye is entrapped inside the nanoparticles by an electrostatic bond between the anionic (acid) dye and the nanoparticles (Figure 1). The FTIR spectra of the nanopartilces, the acid dye (Telon Red M-BL) and the coloured silica nanoparticles are presented in Figure 1. The first spectrum, related to the nanoparticles shows a characteristic broad band corresponding to hydroxy stretching $(\mathrm{Si}-\mathrm{OH})$ at approximately $3426 \mathrm{~cm}^{-1}$, another characteristic broad band appears at approximately $1094 \mathrm{~cm}^{-1}$ for the $\mathrm{Si}-\mathrm{O}-\mathrm{Si}$ asymmetrical 


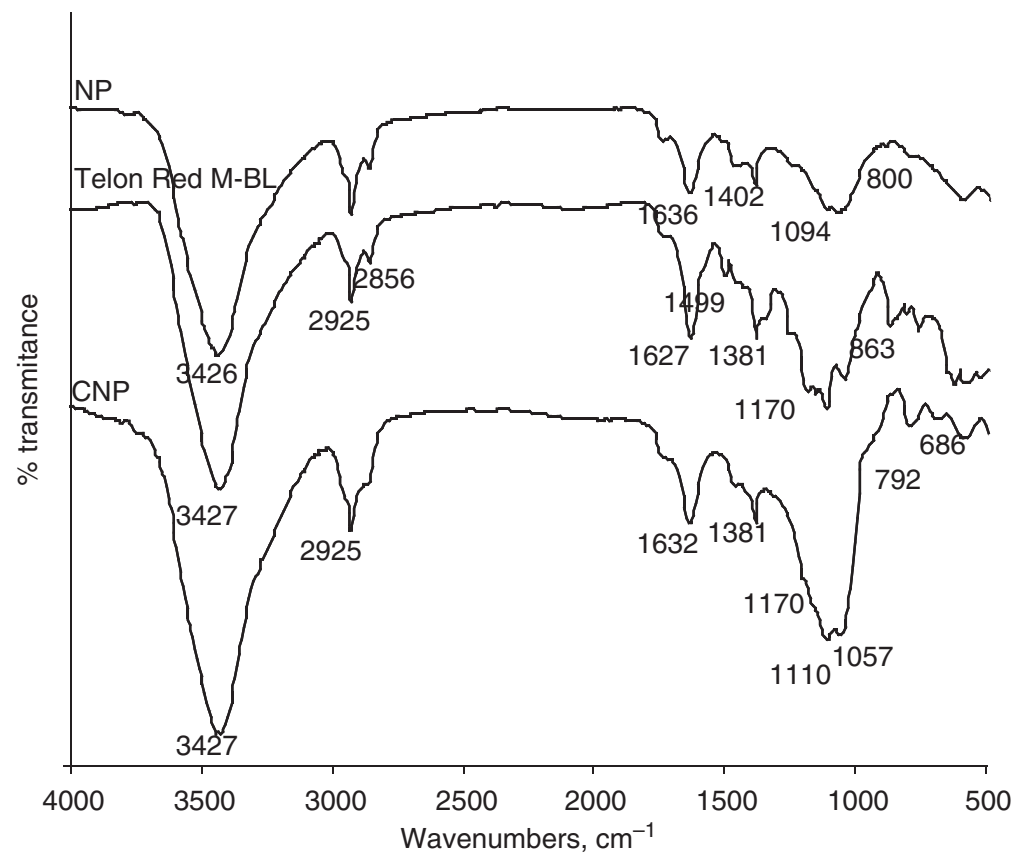

Figure 1 FTIR characterisation of prepared coloured silica nanoparticles

stretching mode and at approximately $800 \mathrm{~cm}^{-1}$, corresponding to the $\mathrm{Si}-\mathrm{O}-\mathrm{Si}$ bending frequencies.

In the Fourier Transform-infrared spectrum of Telon Red M-BL (Figure 1), characteristic broad bands responsible for hydroxy, amino stretching were observed at approximately $3427 \mathrm{~cm}^{-1}$. Aromatic and alkyl C-H stretching vibrations were found at $2925 \mathrm{~cm}^{-1}$ and $\sim 2856 \mathrm{~cm}^{-1}$, respectively. The peaks at 1627 and $1499 \mathrm{~cm}^{-1}$ were assigned to aromatic $\mathrm{C}=\mathrm{C}$ stretching vibration. At $1499 \mathrm{~cm}^{-1}$ the absorption band was assigned to the $\mathrm{N}=\mathrm{N}$ group. One other peak of this compound appeared at $1381 \mathrm{~cm}^{-1}$ and was attributed to the $v(\mathrm{C}-\mathrm{N})$ mode, corresponding to the amino group on the aromatic ring. The absorption bands of asymmetric and symmetric stretching of the $\mathrm{O}=\mathrm{S}=\mathrm{O}$ groups lie at approximately $1350 \mathrm{~cm}^{-1}$ (hide) and $1170 \mathrm{~cm}^{-1}$, respectively, corresponding to the $\mathrm{SO}_{3} \mathrm{H}$ characteristic peaks of the acid dyes. The absorption band of the stretching vibration of the $\mathrm{S}-\mathrm{O}(\mathrm{H})$ in the $\mathrm{SO}_{3} \mathrm{H}$ groups lie at approximately $863 \mathrm{~cm}^{-1}$.

The Fourier Transform-infrared spectrum of synthesised coloured silica nanoparticles shows characteristic bands of nanoparticles and Telon Red M-BL, with some modifications promoted by immobilisation of the dye to the nanoparticles. New bands are shown when compared with the nanoparticle spectrum; namely, characteristic bands corresponding to the $\mathrm{SO}_{3} \mathrm{H}$ group $(\sim 1350,1170$ and $863 \mathrm{~cm}^{-1}$ ) and bands between 600 and $800 \mathrm{~cm}^{-1}$, attributed to vibration of $\mathrm{C}-\mathrm{H}$ from the aromatic ring in the acid dye, suggesting the presence of the acid dye in the nanoparticles.

Figure 2a is a transmission electron microscopy image of the coloured silica nanoparticles, which are spherical in shape and $\sim 150-250 \mathrm{~nm}$ in diameter. The particle size distribution of the obtained coloured silica nanoparticles according to dynamic light scattering is shown in Figure 2b: the presence of two distinct peaks indicates the presence of a mixture of small and big particles (a mixture of 145- and 489-nm particles), and the average particle size was found to be $206 \mathrm{~nm}$. However, from transmission electron microscopy investigation, the large particles detected by dynamic light scattering should arise from aggregates.

\section{Hair dyeing}

Coloured silica nanoparticle diffusion into hair fibres Diffusion of coloured silica nanoparticles into hair fibres (dyeing) has been investigated. On the one hand, coloured silica nanoparticles do not penetrate at all, or do so only sparingly into the structure of Caucasian virgin hair. They seem to have difficulty in penetrating hair. It is possible that these particles do not fit into the fibre's cell membrane complexes, which are known to be the diffusion pathways in the keratin fibre [7]. On the other hand, coloured silica nanoparticles diffused readily into bleached hair fibres (swelling hair), using the same dyeing conditions. Chemical bleaching with alkaline peroxide not only alters melanin, but also destroys some disulphide bonds from the cuticle region to the centre of the cortex region of the hair, which leads to a breakdown of the cystine linkages and hydrolysis of the peptide chains to form shorter chains with the thiol groups. It weakens the cell membrane complex hydrolysing the thioester linkages, which bond the lipids to the protein surface; the underlying proteinaceous surface reveals a variety of functional groups available for interactions with novel molecular and nanoparticles entities. The bleaching reaction results in the breakdown of the cell membrane complex, the cuticle and cortex components, making the hair fibre swollen, full of cavities (holes) and causing the cuticle to rise [10]. Scanning electron microscope images revealed that the cuticle and the cortex of the bleached hair were swollen and full of holes (Figure $3 b$ ) contrasting that of virgin hair (Figure 3a). 

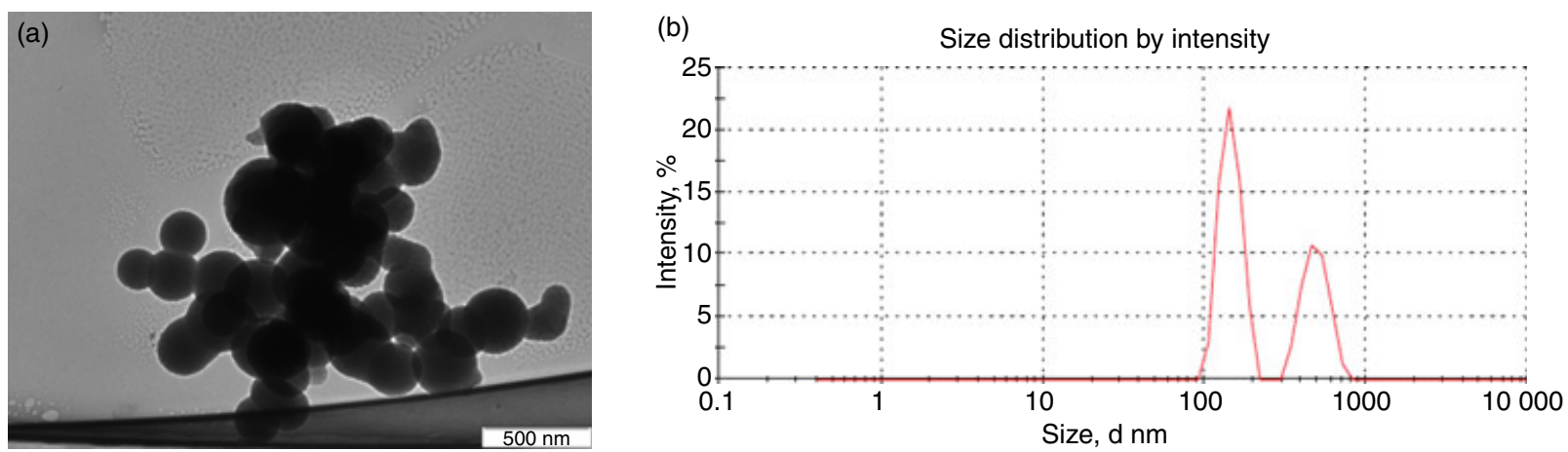

Figure 2 (a) TEM image of the coloured silica nanoparticles; (b) dynamic light scattering data of coloured silica nanoparticles

The diffusion of coloured silica nanoparticles into bleached hair was optimised at low $\mathrm{pH}$. Coloured silica nanoparticles are adsorbed first at the surface of the bleached hair fibres; these coloured silica nanoparticles may attach to hair via electrostatic linkages between the cationic, protonated amino end groups of the hair $\left(\mathrm{NH}_{3}{ }^{+}\right)$ and the anionic sulphonate groups of the dye (dye- $\mathrm{SO}_{3}^{-}$) entrapped on the silica nanoparticles and/or the anionic silanol groups of the silica nanoparticles $\left(\mathrm{Si}-\mathrm{O}^{-}\right)$. A preferential uptake of the coloured silica nanoparticles (white dots) at the cuticle scale edges can be seen (Figure 4). Adsorption of aggregates of coloured silica nanoparticles at the fibre surface may occur; these nanoparticles have a high tendency to form aggregates.

The coloured silica nanoparticles are then absorbed into the outer cuticle layers of the bleached hair (Figure 5). The scanning electron microscopy picture shows that, apart from the lift-off of the cuticle and the disappearance of some cuticle edges, another noticeable effect is that, just beneath some layers of the cuticle, there is the shape of aggregates of coloured silica nanoparticles. This may be attributable to coloured silica nanoparticles being held underneath the margins of uplifted cuticle scales.

In the final step, some coloured silica nanoparticles diffuse into the hair cortex, where they may or may not subsequently participate in interactions with a variety of

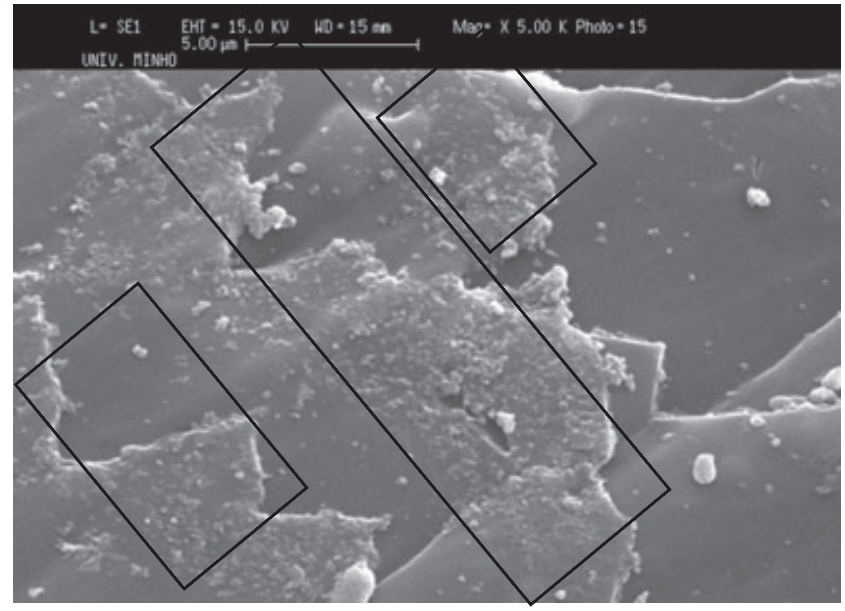

Figure 4 SEM picture of the surface of a human hair dyed with coloured silica nanoparticles $(7.5 \%$ owf $)$ in ethanol/benzyl alcohol $(5 / 5 \% \mathrm{v} / \mathrm{v})$ mixture solvent at $45{ }^{\circ} \mathrm{C}$ and $\mathrm{pH} 4.5$ for $1 \mathrm{~h}$

functional groups available in bleached hair (Figure 6a). The scanning electron microscopy picture of the fibre cross section shows coloured silica nanoparticles distributed mainly in the outer regions of the hair structure (the inner layers of the cuticle and the outer region of the cortex). The coloured silica nanoparticles that do not diffuse from the cuticle to the cortex may participate in interactions with the available functional
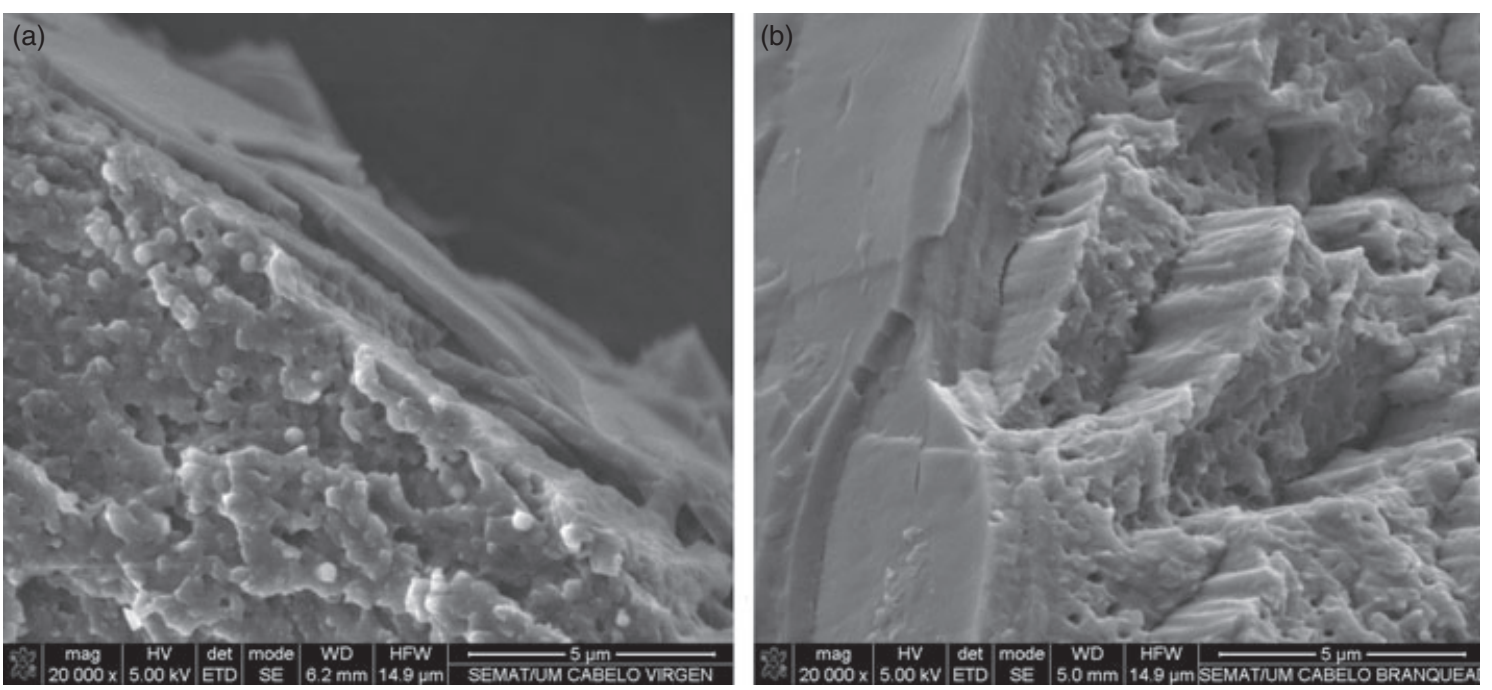

Figure 3 SEM pictures of fibre cross sections of human hair after embedding in resin: (a) virgin hair and (b) bleached hair 


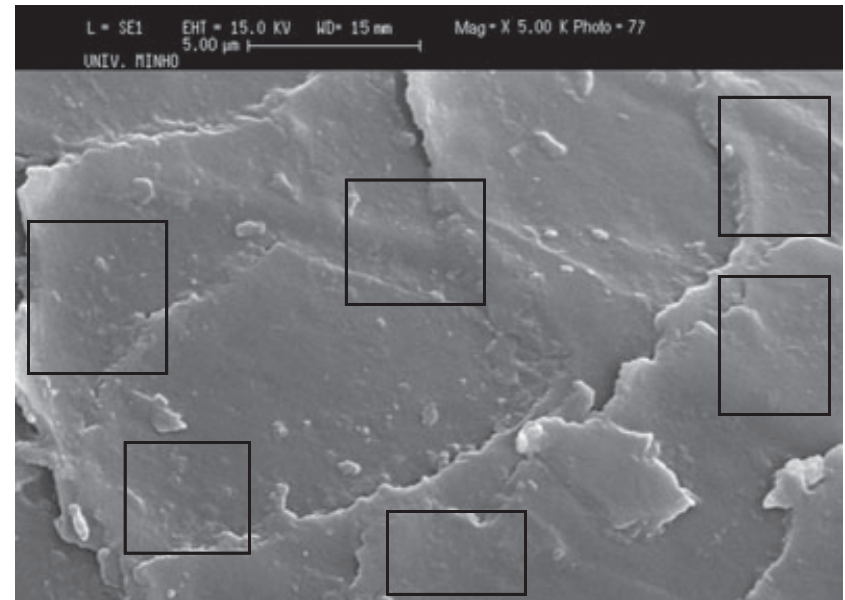

Figure 5 SEM picture of the surface of a human hair dyed with coloured silica nanoparticles (7.5\% owf) in $10 \%$ benzyl alcohol at $45{ }^{\circ} \mathrm{C}$ and $\mathrm{pH} 4.5$ for $1 \mathrm{~h}$

groups at the cuticle layers (Figure 6b) and this could be because the interactions of the coloured silica nanoparticles in the cuticle layers of the fibre with the functional groups of the fibre may be quicker than the diffusion of coloured silica nanoparticles into the interior of the fibre [11]. In Figure 6b, the scanning electron microscopy picture, being at 100000 magnification, easily shows one isolated coloured silica nanoparticle at the cuticle of the hair, with a regular and circular shape characteristic of nanoparticles and a size of $\sim 150 \mathrm{~nm}$, and it shows an irregular hair cuticle's surface. This may be as a result of coloured silica nanoparticles being held underneath the margins of uplifted cuticle scales, supporting the observation made in Figure 5.

After dyeing the hair with coloured silica nanoparticles, the morphological structure of the hair changes. Figure 7 shows a cross-section picture of human hair dyed with coloured silica nanoparticles (Figure 7b), contrasting that of human bleached hair (before dyeing) (Figure 7a). Inside dyed hair, the free volume cavities of the outer regions of the dyed hair structure (the inner layers of the cuticle and the outer region of the cortex) are filled up. Rapid interactions may occur between the coloured silica nanoparticles and the functional groups in the fibre, leading to the formation of bulky, immobile coloured silica nanoparticles, which seem to fill up the free volume cavities. This results in the reduction of the overall size of free volume holes in hair.

According to the literature, two major diffusion pathways into hair fibres exist. The transcellular route describes the pathway across the low and high sulphur parts of the cuticle cells. This pathway is important, especially for small hydrophilic molecules. An alternative pathway is intercellular diffusion, which is preferred for large hydrophobic or apolar molecules, as shown in a study on diffusion into hair by fluorescent dyes [7]. Molecules penetrate the cuticle via low sulphur regions of the cuticle, the intercellular material and the endocuticle. Low sulphur regions can function as preferred diffusion pathways because of their swelling behaviour in aqueous solutions. Our experiments showed that coloured silica nanoparticles do not penetrate into the structure of Caucasian virgin hair. In contrast, coloured silica nanoparticles diffused readily into bleached hair fibres. This observation demonstrates that the intercellular route for the coloured silica nanoparticles diffusion into hair should be the preferred one; i.e. through the nonkeratinous regions of hair, such as the cell membrane complex. The size of the coloured silica nanoparticles may have contributed to the preference for this route.

\section{Shampoo washing fastness}

Figure 8 shows the total colour changes of hair samples dyed with coloured silica nanoparticles in terms of $\Delta E$ values after 13 washes over the control sample. The data show that the most significant colour lost, over the 13 cycles, is during the first six washes (probably because some coloured silica nanoparticles were at the surface of the fibre and were removed during the six washes). After six washes, there is no significant colour lost (which suggests that the coloured silica nanoparticles are trapped inside the hair fibres and not able to come out easily).
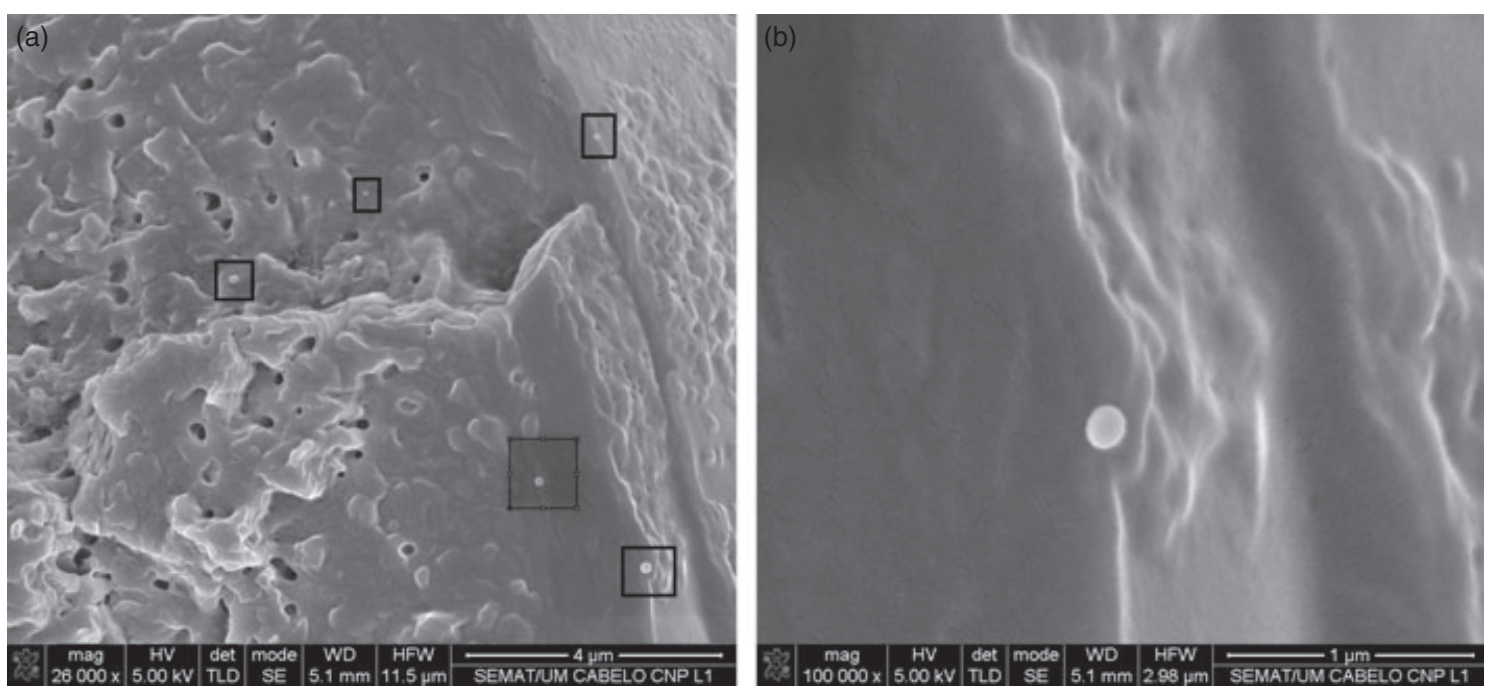

Figure 6 SEM pictures of fibre cross sections of human hair dyed with coloured silica nanoparticles (7.5\% owf) in $10 \%$ benzyl alcohol at $45{ }^{\circ} \mathrm{C}$ and $\mathrm{pH} 4.5$ for $1 \mathrm{~h}$ and embedded in a epoxy resin: (a) 25000 magnification and (b) 100000 magnification 

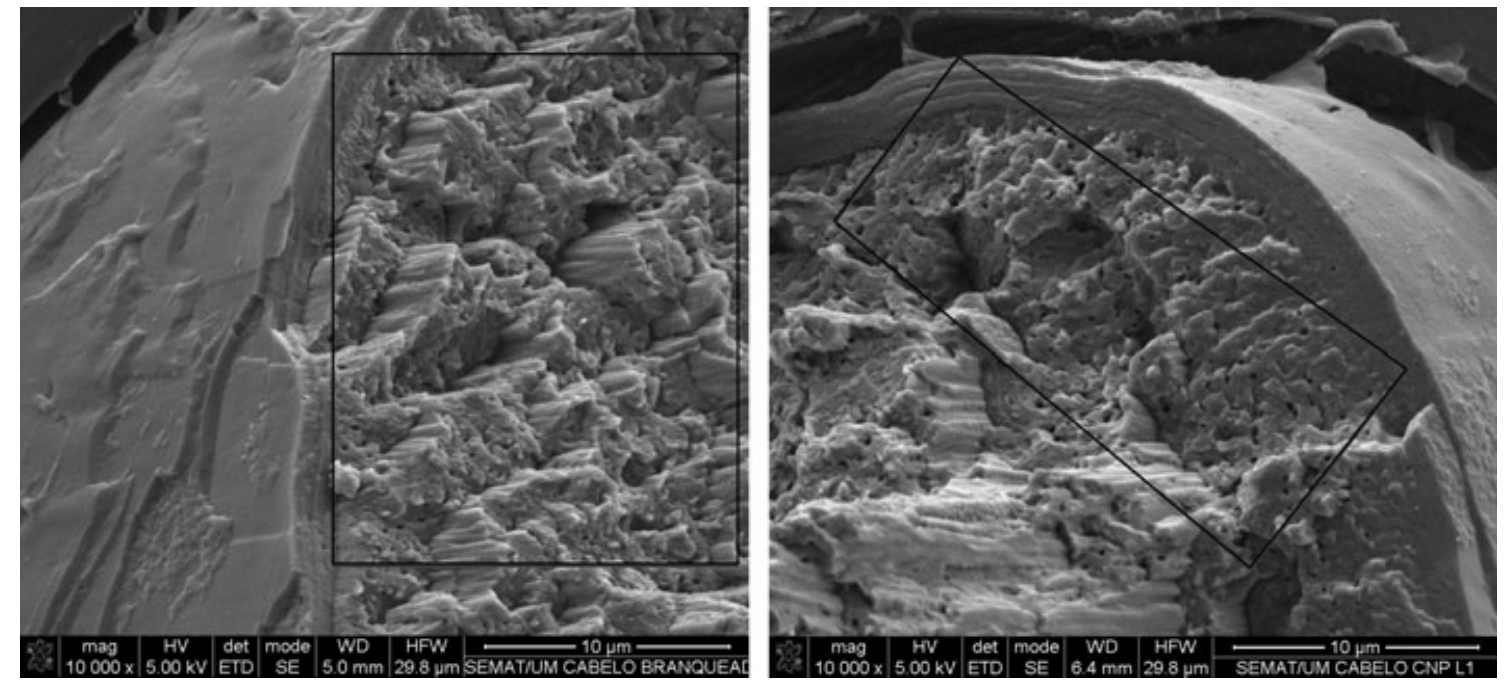

Figure 7 SEM pictures of fibre cross sections of human hair: (a) hair bleached using commercial cream at $40{ }^{\circ} \mathrm{C}$ for 30 min and embedded in a epoxy resin; (b) dyed with coloured silica nanoparticles (7.5\% owf) in $10 \%$ benzyl alcohol at $45{ }^{\circ} \mathrm{C}$ and $\mathrm{pH} 4.5$ for $1 \mathrm{~h}$ and embedded in a epoxy resin

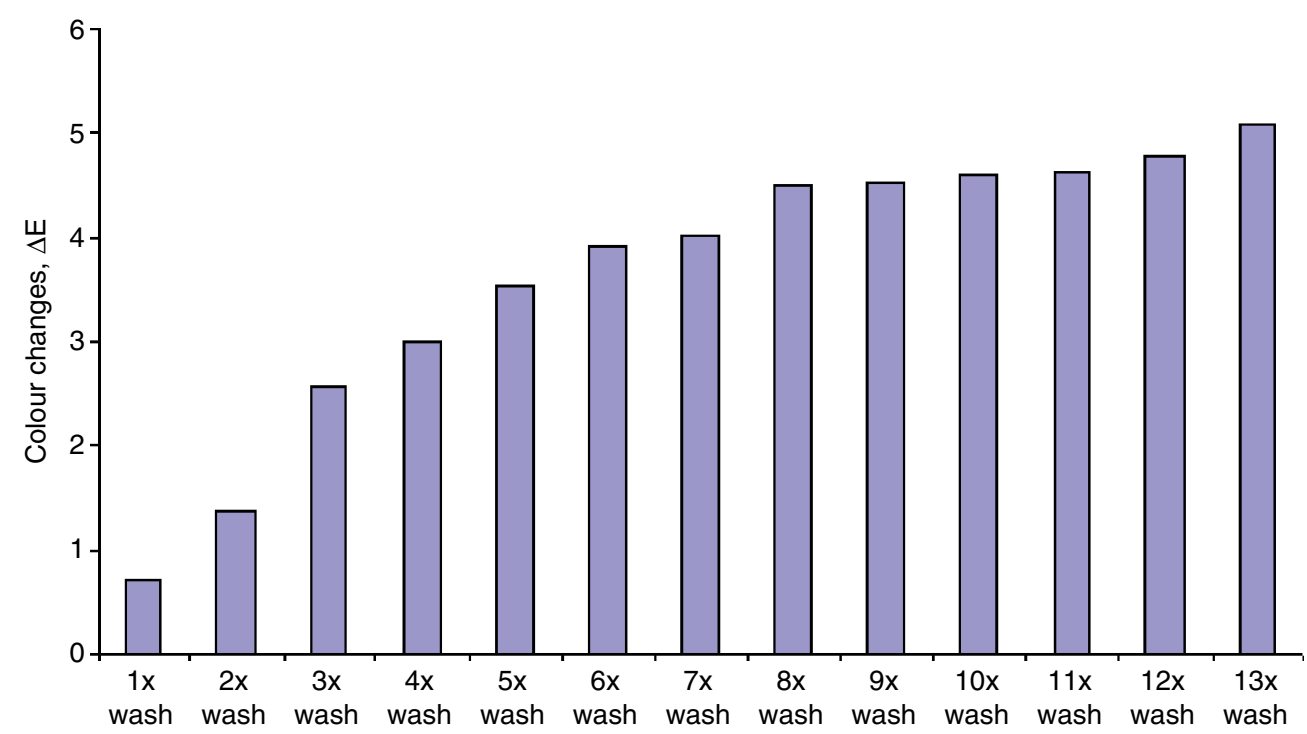

Figure 8 Colour fastness of hair samples dyed using coloured silica nanoparticles

Because of the coloured silica nanoparticle-acquired functional groups (after entrapment of dye), the size and the surface chemistry, hair dyed with coloured silica nanoparticles showed good wash fastness to shampooing up to 10 washes. The difference in $\Delta E$ values can be visually observed [12].

\section{Conclusions}

The present work has led to a novel method of dyeing human hair fibres using coloured silica nanoparticles. The diffusion of coloured silica nanoparticles into bleached hair was optimised at low $\mathrm{pH}$. Coloured silica nanoparticles are distributed mainly in the outer regions of the hair structure (the inner layers of the cuticle and the outer region of the cortex). The intercellular route for the diffusion of the coloured silica nanoparticles into hair is the preferred one; i.e. through the non-keratinous regions of hair, such as the cell membrane complex. The size of the coloured silica nanoparticles may have contributed to the preference for this route. As a result of the coloured silica nanoparticle-acquired functional groups (after entrapment of dye), the size and the surface chemistry, dyed hair showed good wash fastness to shampooing up to 13 washes.

\section{References}

1. J R Gomes, S Sampaio and F Maia, Nanotech 2009, Houston, USA (2009).

2. J Yan, M C Estevez, J E Smith, K Wang, X He, L Wang and W Tan, Nano Today, 2 (2007) 44.

3. F Redlick and J DeKoven, CMAJ., 176 (4) (2007) 445.

4. S J Meade, J M Dyer, J P Caldwell and W G Bryson, Text. Res. J., 78 (2008) 943.

5. L dos Santos and I Joekes, Colloids Surf. B Biointerfaces, 40 (2005) 19.

6. I Seshadri and B Bhushan, Acta Mater., 56 (2008) 774.

7. C R Robbins, Chemical \& Physical Behavior of Human Hair, 2nd Edn (New York: Springer Verlag, 1988) 151.

8. M Fujiwara, K Shiokawa, K Hayashi, K Morigaki and Y Nakahara, J. Biomed. Mater. Res. A, 81A (2007) 103. 
9. W Stober, A Fink, E Bohn. J. Colloid Interface Sci., 26 (1968) 62.

10. C Bolduc and J Shapiro, Clin. Dermatol., 19 (2001) 431.

11. K Schafer, Wool Technol. Sheep Breed., 42 (1994) 59.
12. Difference in DE values (online: http://img153.imageshack. us/img153/795/img0381figpaper13washes.jpg; last accessed, 28 Nov 2010). 